\title{
Implementing TD-LTE as Software Defined Radio in General Purpose Processor
}

\author{
Jussi Kerttula, Nicolas Malm, Kalle Ruttik, Riku Jäntti and Olav Tirkkonen \\ Department of Communications and Networking \\ School of Electrical Engineering \\ Aalto Unversity, Finland \\ \{jussi.kerttula, nicolas.malm, kalle.ruttik, riku.jantti, olav.tirkkonen\}@aalto.fi
}

\begin{abstract}
Cloud radio access networks use servers that are connected to Remote Radio Heads (RRH). Base station (BS) implementation with this concept is challenging. The strict realtime nature of baseband (BB) processing seems to rule out usage of General Purpose Processors (GPP) with non-real time Operating Systems (OS). In this paper, we propose a BS architecture where most of the real-time processing is confined into a Virtual Hardware Enhancement Layer (VHEL). VHEL hides the hardware non-idealities from the software and vice versa. Possible errors due to the non-realtime OS and RRH appear as channel errors, which makes software development easier. We demonstrate the benefits of our architecture by implementing a Time-Division LTE system (TD-LTE) in $\mathrm{C}++$ and running it as a user process in an Intel i7 class PC. Over-the-air transmissions are realized using USRPs. We report the performance of the implemented platform. We observe that with the given VHEL the transmitter and receiver never lose synchronization. Also the PC tends to be quick enough to feed the data; and the loss rate of subframes due to the non-real-time nature of the platform is relatively low. The proposed platform provides the possibility to implement TD-LTE on GPPs and virtual machines.
\end{abstract}

\section{Categories and Subject Descriptors}

C.2.1 [Network Architecture and Design]: Wireless Communication

\section{General Terms}

Design, Experimentation, Algorithms

\section{Keywords}

SDR programming model, SDR architecture, prototyping

Permission to make digital or hard copies of all or part of this work for personal or classroom use is granted without fee provided that copies are not made or distributed for profit or commercial advantage and that copies bear this notice and the full citation on the first page. Copyrights for components of this work owned by others than ACM must be honored. Abstracting with credit is permitted. To copy otherwise, or republish, to post on servers or to redistribute to lists, requires prior specific permission and/or a fee. Request permissions from permissions@ acm.org.

SRIF'14, August 17-22 2014, Chicago, IL, USA.

Copyright 2014 ACM 978-1-4503-2995-8/14/08 ...\$15.00.

http://dx.doi.org/10.1145/2627788.2627793.

\section{INTRODUCTION}

Software Defined Networking (SDN) has emerged as a new paradigm of network development. SDN provides network owners and developers with open, programmable communication stacks and has changed the way we think about core network design. The development of SDN is driven by the benefits that common purpose servers provide as compared to specialized hardware. Software Defined Radio Access Networks (SDRAN) [10] and Cloud based Radio Access Networks (C-RAN) [9] [15] bring the same benefits into radio access network design. A flexible, open and programmable SDRAN could significantly reduce network operation and maintenance costs. Close to the air interface radio network units, Base Stations (BS) and User Equipment (UE) have very tight timing requirements. The traditional embedded real-time implementations of these do not seem to fully support a software defined model of RAN.

The time constraints of RAN are imposed by the timings at the radio interface. In the base band (BB) processing side the stream of $\mathrm{A} / \mathrm{D}$ samples can be collected into blocks that are sub-sequentially processed by a high speed processor. Such temporal decoupling of sampling and processing is a natural way to implement wireless signal processing on a general purpose processor [18]. This idea has been commercialized by Vanu incorporation [7]. The architecture envisioned in [18] virtualizes the remote radio head. Such virtualization is a basis for many software defined radio (SDR) environments such as GNU Radio [8], IRIS [16] and ASGARD [5]. These environments provide software platforms where wireless signal processing algorithms operate on blocks of samples collected by some peripheral equipment. One commonly used radio frontend peripheral is Universal Software Radio Peripheral (USRP) [12]. USRP products have become popular in research due to their relatively low cost, good community support and flexibility.

Existing SDR environments are inclined towards data flow type signal processing. These environments are suitable for serving physical layer algorithms but are cumbersome when developing higher layer protocols that usually function as state machines. Projects that aim for implementing the full communication stack such as OpenBTS [13] and OpenLTE [14], use SDR tools only for physical layer implementation. In these projects higher layers are implemented in the $\mathrm{C}++$ programming language without SDR tools.

The higher layers would also benefit from specialized development tools. Early attempts with such tools produced executable code but the code was somewhat detached from the physical system implementation. For instance SDL based 
tools [6] generate codes that can be used for logical algorithm testing but are not directly applicable to production level systems. A more practical code requires tighter integration between protocol generation tools and underlying hardware. The TRUMP project from Aachen University has demonstrated such an environment. TRUMP is a protocol generation tool that operates on a programmable WARP SDR platform [19].

In this work we illustrate how the tight real time processing of analog-to-digital (A/D) converters and the processing in SDR protocol stack can be separated. Such separation requires addressing of tight timing related constraints. In SDR platforms the timing is handled by implementing time critical functions on specialized hardware [20][11][17]. In [20] for instance the timing is handled by GPS and some time critical functions are handled by specialized hardware. Similarly in [11] an LTE type BS implementation is reported that collects A/D (D/A) samples from specialized hardware and time critical functions are hand tuned. However, the Amarisoft implementation [4] demonstrates that general purpose processors can serve as a LTE BS without a need of specialized hardware. Due to its closed source nature little is known about this [4] implementation.

In this paper we report an SDR implementation which decouples the $\mathrm{BB}$ processing and radio transmission from the remote radio head $(\mathrm{RRH})$. The architecture and optimization of GPP-based soft LTE BS is discussed previously in [17]. The implementation in [17] reports multiple realtime processing and optimization related issues. Our implementation alleviates some of these reported issues. We have implemented a virtualized hardware enhancement layer (VHEL) that hides RRH specific operations from the BB processing. A most important feature of this layer is virtualization of the RRH hardware. The VHEL hides possible delays overflows and underflows from BB processing. With help of VHEL we can operate a Time-Division Long-Term Evolution (TD-LTE) BS stack in a non-real-time operating system.

Our system contains a TD-LTE BS (and UE) operating on a GPP and USRPs (N200s and N210s) radio units 1. All the $\mathrm{BB}$ processing is handled by GPP and the RRHs only perform analog-to-digital, digital-to-analog conversion and Radio Frequency (RF) processing. The software is written in the $\mathrm{C}++$-programming language. Our platform is useful for testing new radio features, building proof-of-concepts, and for teaching and learning purposes. We propose an architecture for software defined RANs. We describe the structure of the platform, discuss some of the challenges we have faced and present measurement results. The VHEL maintains packet synchronization, sample synchronization and mitigates - to the extent feasible - the impact of lost or late BB packets. Thanks to these functions we have effectively eliminated the strict real-time requirements from $\mathrm{BB}$ operations. Even if the BB processing is late the system will continue to operate. Our architecture is similar to the virtual base station pool architecture suggested for WiMAX in [20] which also relies on GPP and a non-real-time operation system for $\mathrm{BB}$ processing. The main differences between [20] and our work is that we use over the air synchronization that is implemented not in special hardware but in PC. We also focus on TD-LTE instead of WiMAX. LTE has more stringent timing constraints than WiMAX. Instead of $5 \mathrm{~ms}$

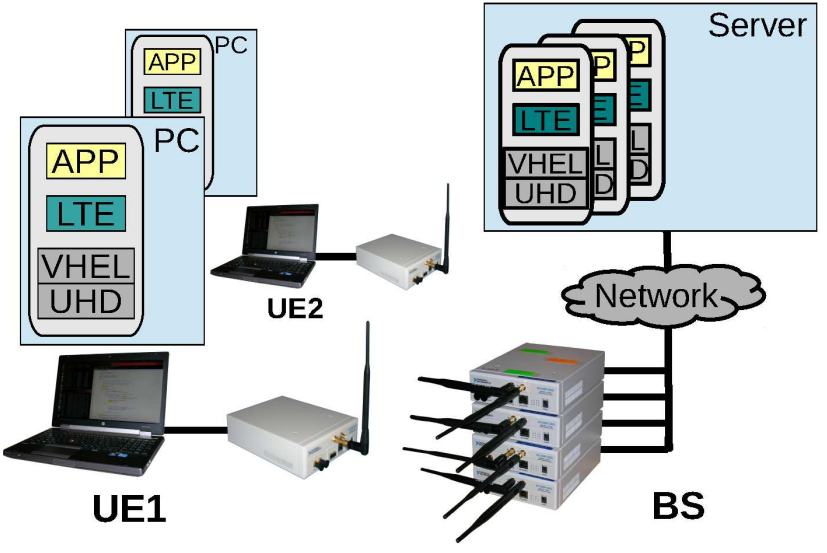

Figure 1: Figure of the test-bed (multiple tx rx with usrp + PCs).

processing delay reported in [20] our system handles one subframe in every $1 \mathrm{~ms}$.

\section{SDR BASED RRM TEST-BED}

The testbed is based on implementation of a TD-LTE system according to 3GPP specifications TS-36.211, TS-36.212 and TS-36.213 [2, 1, 3]. We implemented RLC/MAC/PHY functions required for encoded transmission over the air data. The implemented radio stack is visualized on Fig. 2. The platform is written in $\mathrm{C}++$ and consists of nodes comprised of a USRP (either N200 or N210) and a PC for baseband processing. The code can run as either a BS or UE on a standard non-real-time (non-RT) Linux OS.

Our code demonstrates that complex functions such as a BS or a UE can operate successfully on such a platform. Operation was tested on Ubuntu 12.04 with stock kernel. The system can run with $1 \mathrm{~ms}$ subframe precision and sampling rates as specified in the standards. We have tested the system in $1.4,3,5$ and $10 \mathrm{MHz}$ bandwidths. The system supports all the LTE Release 8 TDD UL/DL subframe configurations. These can be changed on the fly by simply changing system configuration parameters. In conventional approach such systems are implemented using both custom dedicated hardware, and a tailored RT operating system. This is to guarantee timing of execution and power efficiency through careful tuning. The key of our solution to providing a similar level of performance - but with greater flexibility - lies in the special architecture we have developed. It aims to deal with and hide the implementation issues related to a non-RT OS platform.

The software follows a traditional layered structure. Most of the real-time related issues and non-idealities related to RRH are confined to VHEL. This allows LTE platform developers to focus on protocol and standard related issues. Sending samples to and receiving samples from $\mathrm{RRH}$ (in this case, a USRP) is the part that poses the real-time requirements and restrictions. We hide those constraints as much as possible from the BB processing without losing the ability to run a fully functional LTE base station. As a result, development of higher-layer $\mathrm{BB}$ processing resembles simulator development. The platform enables a development model where the USRPs are replaced with a UDP/IP connection to a radio simulator. This enables rapid develop- 


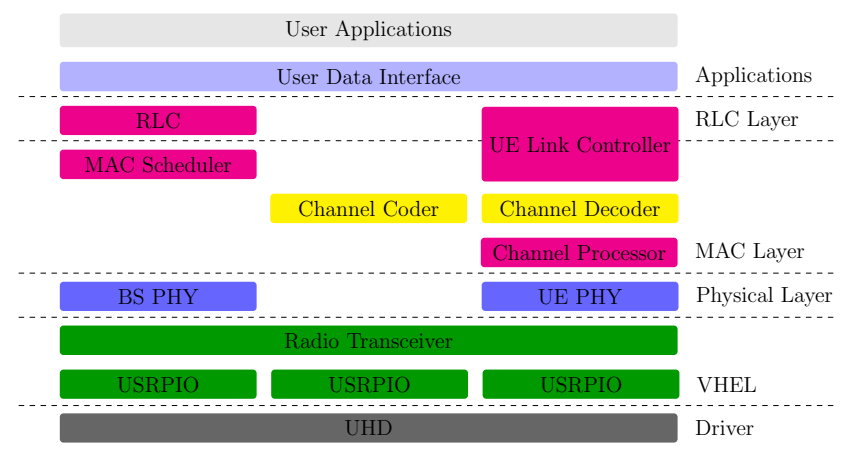

Figure 2: Layer-wise Overview of Components

ment and testing. The same code can then be recompiled to run on the hardware devices. Furthermore, VHEL enables virtualized execution of the code. Multiple virtual machines on the same physical host may run multiple BSs (or UEs), each with one or more dedicated radio heads.

Both BS and UE provide PSS, SSS, PFICH, PHICH, PD$\mathrm{CCH}, \mathrm{PDSCH}, \mathrm{PUCCH}$ and PUSCH. Details of these channels can be found in the specification TS-36.211 [2].

\section{CHALLENGES OF RT OPERATION}

Running real-time baseband processing on a non-real-time GPP computer - or even a virtual machine - requires new architectural solutions. Here, we report some of the practical limitations we have observed. Most of the real-time constraints relate to the synchronous nature of the radio transmissions.

We can group the challenges into three main categories: clock skew, unreliable link between the PC and RRH, and latency between the PC and RRH. In what follows, we discuss these challenges in detail, and in Sections 4 and 5 we describe how these issues are addressed in our architecture.

\subsection{Local oscillator inaccuracy}

TD-LTE expects that the transmitter and receiver have synchronized to have the same clock. Remote radio heads have imperfect local oscillators (LO). These inaccuracies affect the carrier frequency and sampling frequency. USRP N200/210 local oscillators are temperature-compensated crystal oscillators (TCXO) with an accuracy of $2.5 \mathrm{ppm}$ (parts per million). To put this into perspective, at a carrier frequency of $1 \mathrm{GHz}$ the offset is $\pm 2.5 \mathrm{kHz}$ whereas the LTE standard mandates subcarrier spacing to be $15 \mathrm{kHz}$. In OFDM systems such as LTE, even small carrier frequency offsets cause inter-carrier interference (ICI). The more expensive NI USRP-2932 incorporates an integrated GPS disciplined clock that provides significantly better accuracy. Even without GPS the LO accuracy is $2.5 \mathrm{ppb}$ (parts per billion), meaning that at the same carrier frequency of 1 $\mathrm{GHz}$ the offset is only $\pm 2.5 \mathrm{~Hz}$. This is accurate enough to keep ICI low, but still needs to receive occasional frequency corrections that can handled by the equalizer.

The local oscillator also drives sampling. The difference in sampling rate is visible as a complex signal phase error, and if the error is large enough the receiver and transmitter can have differing number of samples. With an oscillator accuracy of $2.5 \mathrm{ppm}$ and $5 \mathrm{MHz}$ LTE bandwidth it means every $40 \mathrm{~ms}$ the transmitter and receiver see a one-sample difference. With $2.5 \mathrm{ppb}$ the difference appears every $80 \mathrm{~s}$.

In this paper we proposed a VHEL that handles the timing errors without traditional phase lock loops. The tested VHEL operates with USRP N200 units and we observed received signal SNR in order of $17 \mathrm{~dB}$.

\subsection{Lost data}

Software radios can encounter packet loss for various reasons. On the transmitter side, the processing might just not have been quick enough to prepare the packet in time. This could be due to slow code, OS scheduling problems or simply a processor that is not fast enough. When transmission time arrives without the $\mathrm{BB}$ components being ready in time, it results in missing the transmission opportunity for the subframe in question. In a properly designed real-time embedded system such a situation should not occur due to timing guarantees. If a system can produce the data in time once, it can repeat the feat every time. Another possible source of packet loss is connection between RRH and SDR. In our exemplary implementation we have a Gigabit Ethernet link connecting the USRP and the host computer. The driver uses UDP to transfer sample data over it. As in any network, these UDP packets could be lost. Once again, an embedded system do not suffer from this issue since their $\mathrm{A} / \mathrm{D}$ and $\mathrm{D} / \mathrm{A}$ converters connect directly to the BB processor ensuring such losses never occur. Remote radio heads however, required handling of this issue in a manner that shields the rest of the BB processing infrastructure from it.

\subsection{Latency}

Another effect of the network link between RRH and computer is the added latency (compared to the traditional approach) in both the TX and RX chains. In bi-directional communications each received message must be responded to within a certain time limit. In TD-LTE, the shortest feedback window can be 4 subframes $(=4 \mathrm{~ms})$. This means that from the time when the last sample of a subframe is received, we have $3 \mathrm{~ms}$ to prepare a subframe with feedback (e.g. ACK/NACK) to be transmitted in reply. Based on our brief ping-tests and experiments made in [1] the oneway hardware delay between USRP N200/210 and a PC is over $0.5 \mathrm{~ms}$. Most of this stems from to the processing delays in the Gigabit Ethernet stack at both endpoints. This poses a challenge since the round-trip-time (RTT) reduces processing time, leaving only $2 \mathrm{~ms}$ for all computations.

\section{VIRTUAL HARDWARE ENHANCEMENT LAYER}

In general the software RAN design is concentrating on optimizing the code such that it can meet strict real time requirements [20] [17]. Synchronization in these systems is done by using brute force approach. In [20] the interface to $\mathrm{RRH}$ is implemented in FPGA and network synchronization is assisted by GPS signals. The proposed designs demand high precision from baseband processing. The system designer has to identify the performance requirements of each individual process and allocate the resources accordingly. Even with this precise tuning, the platform in [20] is not always capable to cope with the timing constraints.

We propose to handle the problems described in the previous section through the use of a virtual hardware enhance- 


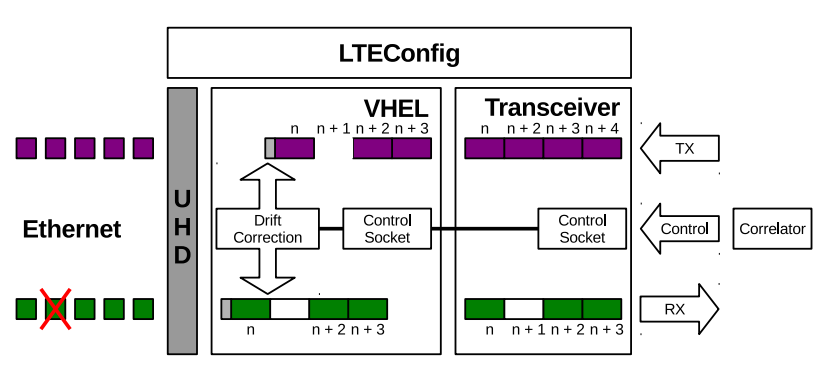

Figure 3: TX and RX chains with VHEL

ment layer (VHEL). It serves to hide all non-idealities offering a more consistent interface to BB processing thereby effectively isolating it from these issues. The operations of VHEL are illustrated on Fig. 3. The VHEL handles sampling alignment, burst sending and reception as well as lost subframes, so that $\mathrm{BB}$ processing components always see the expected number of subframes containing the expected number of samples. This design enables us to relax the real-time requirements of higher layer components. They still face some processing speed constraints but no particular handling delay limit is required since VHEL will hide late subframes by sending dummies to maintain frame alignment. The operation of VHEL is described by pseudo-code in Algorithm 1. The VHEL continuously tracks a timing shift and adjust the initial sampling moment to match the shift. The tracking can follow whatever transmitters. For instance it can be used for over the air synchronization of multiple base stations.

While conceptually system independent, our current VHEL implementation possesses many characteristics heavily geared towards the TD-LTE protocol. LTE is very demanding system and the ability to handle it provides confidence that the designed VHEL can be extended to other systems as well.

\subsection{Timing and TX/RX Multiplexing}

The VHEL's LTE specificity is most apparent in the TX/RX handling pattern. The VHEL maintains separate TX and RX queues and provides to higher layer illusion of steady parallel flows of complete subframes. In order to meet the 1 ms timing constraint of LTE subframes the actual RX subframe is received from USRP in two parts. First we receive half of samples the RX subframe then we send TX subframe and continue with received rest of the RX subframe. Interleaving the TX sample sending provides half-a-subframe's worth of extra time to ready the outgoing burst while not risking the subframe being late at the USRP as would be the case by transmitting at the end of the timing window.

The whole VHEL stack's timing derives from the constant inbound sample stream from the USRP. The clocks of transmitting and receiving USRPs run in different speed and over the time that leads to different number of samples at TX and RX. A LTE base station transmits continuous stream of SSS and PSS synchronization sequences. Our receiver higher layers continuously tracks the correlation with those sequences. If drift regarding the expected moment is identified a correction information is sent to VHEL. VHEL adjusts sampling moment by adding or dropping number of samples.
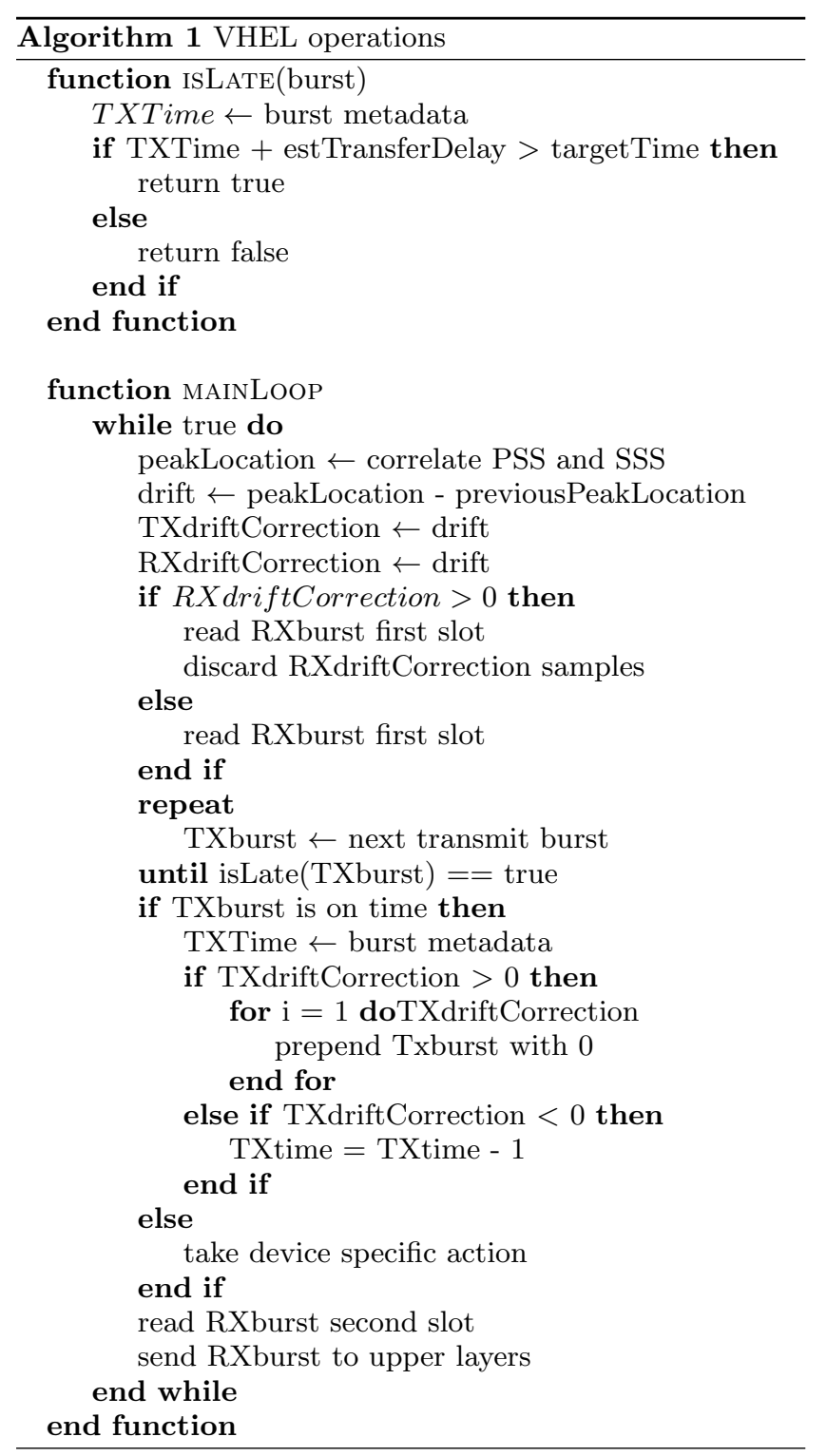

\subsection{TX Flow Management}

VHEL matches the continuous clock of A/D (D/A) converters and packet based $\mathrm{BB}$ processing. In RRH the samples are transmitted and received with constant rate. The transmitted samples of a subframe are processed in packet and this packet has to be in RRH when transmission starts. The received samples are collected into packet and the full packet is send to PC.

The sample flow provides the clocking of the system. VHEL alternates between TX and RX buffers and takes care that the packets are received and transmitted in time. When the VHEL waits for packets from RX (or TX) processing of the packets in other direction are blocked. In transmitter side it waits for the TX subframe from the BB processing upper layers. It can not wait indefinitely since the samples of the subframe have to be in RRH at the transmission moment.

The VHEL ensures that the TX packets are transmitted in right slots of the frame. If the TX packet is late the VHEL can forward to RRH a filling packet of zeros or "contingency subframes". Responsibility for the generation such 
"contingency subframes" would lie with the upper layers as they would be protocol specific. If the late packet still arrives into buffer the VHEL drops the packet and keeps track the timing such that the transmission timing of the next subframe is correct.

By dropping the late packets we reduce reliability of the channel. However, in radio channels the data can be lost due to the fading and communication systems have in-build ARQ mechanism for handling disappearing subframes. Therefore, whether the loss was due to unfavourable channel conditions or the transmitter's inability to produce the required data in a timely manner is indistinguishable to the LTE functions. Retransmissions will operate normally, freeing the VHEL from the need to maintain protocol integrity.

\subsection{RX Flow Management}

On the receiver side, the VHEL reads in the expected number of samples for a subframe at each iteration. It may also read extra samples or discard some to correct for the drift between local oscillators on the local and remote devices. The VHEL also tracks the associated timestamps received from the USRP device through the driver. Continuity tracking helps to detect cases of lost UDP packets. When such an event occurs zeros are forwarded to the upper layers.

\subsection{Sample Level Synchronization}

The VHEL hides differences in clock between the LO and the remote devices from BB processing in two different ways. The clock difference produces carrier frequency errors and sampling rate errors. The carrier frequency errors are neutralized by equalizer. The sampling errors can't be corrected without external help since the VHEL lacks a frequency estimator and/or phase locked loop (PLL) to provide clock adjustment. We use the Primary and Secondary Synchronization Signals (PSS and SSS) from TD-LTE to identify packet and sample timing moment. The peak of PSS, SSS correlator output is compared to expected timing moment. The difference is the estimated of the sampling moment shift due to the sampling clock error. The correlator is implemented in $\mathrm{BB}$ processing. The drift estimator output is feed back from BB to VHEL. The VHEL adjust the TX and RX sample stream such that the drift is removed. After drift correction the VHEL provides the BB processing with aligned subframes with minimal time skew and small phase differences. Furthermore, since VHEL maps for one radio frontend and acts autonomously, the approach allows to support multiple radio frontends each with its specific drift correction. It is a natural method for providing operation with multiple time aligned $\mathrm{RRH}$.

\section{BASE BAND PROCESSING WITH VHEL}

The VHEL handles real-time radio transmission related issues on the radio interface. In an ideal system the BB processing can operate as a simulator without any real time constraints. In practice the $\mathrm{BB}$ still has to prepare data in each subframe (1 $\mathrm{ms}$ in TD-LTE system). The VHEL only relaxes the stringent real time constraints. In embedded systems the BB processing has always to be on time whereas in a VHEL mitigated system the BB processing has to be on time only most of the time. If it is late the VHEL hides the lost packets and the system can continue to operate as if the packet was lost in the radio interface. Essentially the $\mathrm{BB}$ processing being late degrades the channel quality.
Maintaining a bidirectional frame structure and alignment presents numerous additional challenges when attempted on a GPP. These can be divided roughly into two categories: non-deterministic sequencing and timing variability. When dealing with a complex protocol such as LTE it becomes necessary to split processing into multiple threads of execution in order to meet processing speed targets. This however introduces uncertainty in how the operating system's scheduler will queue each thread. Since no information about the intended and/or optimal sequence of execution is available to it, it may - and regularly will - choose wrong. This results in performance variability visible as clustering of late subframes when the thread holding up processing does not receive $\mathrm{CPU}$ time. Cset is a command line utility to create and manipulate CPU sets. Through their use one can dedicate certain cores to one or more processes. This eliminates both execution time and cache contention normally associated with default scheduling. Faced with differing numbers of ready bursts an SDR platform must ensure the correct flow of data to the radio frontend in terms of both sequence and rate. The same need to ensure continuity applies on the receive side. Holes in the data stream appear when packets fail to arrive to the baseband processing functions. This may occur due to dropped Ethernet frames or overflown socket buffers.

\section{PERFORMANCE MEASUREMENTS}

In this section we report measurements results of subfarmeloss rate, and sample synchronicity of the system. The VHEL only masks the real time requirements from the BB processing. In order not to degrade the compound channel quality too much, the BB processing has to be on time most of the time. One can expect that on quick enough GPPs the system finishes processing in given time window most of the time. The rare cases when it can not do it will be handled by VHEL.

We have tested the performance of the implemented TDLTE communication stack with different system parameters. The system uses USRP N200 units as RRH and two different host computers. The BB processing PC were using either Intel Xeon E3-1230 or Intel i7-4930K processors. The system can maintain a connection over extended periods of time. The VHEL proved able to cope with both lost and late subframes as well as to keep sample synchronization. We measured the system ability to keep sample level synchronization. The UE synchronization code locates and tracks the PSS and SSS signals from the BS. These are designed to be easily detected and to enable reliable timing alignment maintenance. When run on a GPP however, the lost subframes puncture also the PSS and SSS signals. Our platform is insensitive to sporadic loss of synchronization signals. The synchronization information is accumulated over multiple frames. Loss of a few instances of the synchronization signals is averaged out.

We validated the stability of the VHEL by measurements of over 700 s. During that time the connection was never lost and the system was able to keep sample level alignment between transmitter and receiver. In this time the correction signal was issued 395 times. The frequency or sampling correction signal is mainly related to the stability of the USRP clocks. The implementation is a proof of concept of running time critical system with rather moderate radio frontend and local oscillator quality. 


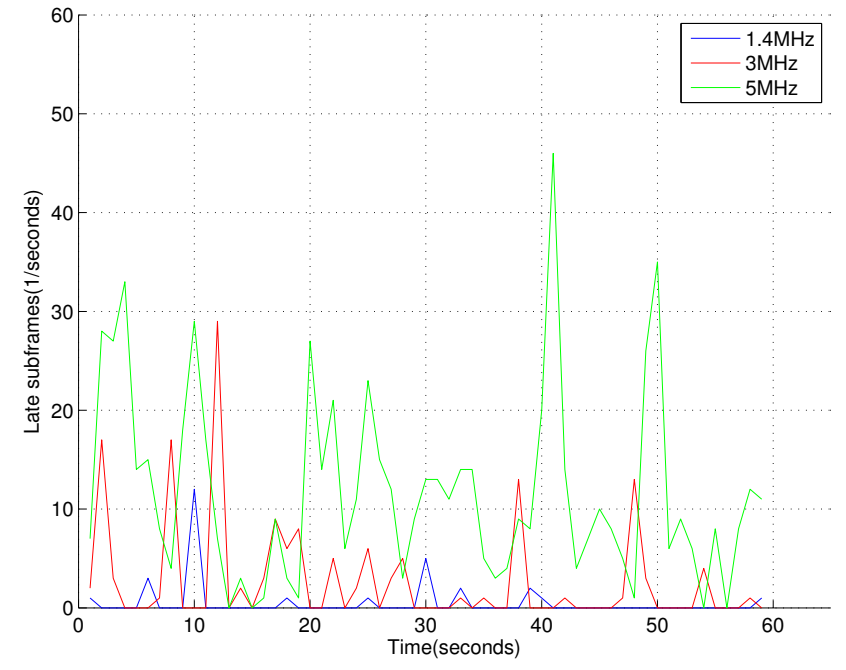

Figure 4: Late subframes per second with E3-1230.

One characteristic of the VHEL is to hide the non-realtime nature of the $\mathrm{BB}$ processing. With fast enough processors one would expect that none of the TX packets will be late. How well the processor will cope with timely processing requirements depends on the capacity of the central processor and the required throughput of processed bits per subframe; in other words the bandwidth of TD-LTE system.

We compared software performance on two host computers. As a performance metric we used the number of late subframes with a duration of one second. During this interval, a TD-LTE system transmits 1000 subframes. A subframe is considered late when the VHEL does not receive it from the upper layers in time to be transmitted.

A measurement over 60 seconds is illustrated in Fig. 4. As one can see, for higher bandwidth the system has more late subframes. These are clustered; meaning that if one is late, with high probability the next ones will also be late. This phenomenon is partly explained by the behavior of the OS scheduler. Since we do not pose any real time constraints on it, the scheduler changes the order of processing threads at will. Sometimes the selected order is unsuitable for the BB processing. Further work has to be done tuning scheduler parameters to be more favorable for the specific workload. For low enough bandwidth the system drops very few frames. In Figure 6 we illustrate the CDF of dropped packets during one second i.e. the subframe loss rate. Level 0 means that no packet was late during the measured second. The Fig. 5 also shows the results when cset is used. With cset one can dedicate certain cores to one or more processes. Since our platform code's performance is not bounded by computation speed but rather by the ability to execute functions in a timely manner, the trading-off of cores against a reduction in context switches proves worthwhile. For the faster computer (with an i7-4930K processor) the system is quick enough to support even $10 \mathrm{MHz}$ TD-LTE bandwidth. In $95 \%$ of the measured seconds no subframe was late for transmission. It should be stressed that the current code is not yet optimized for performance. We have concentrated on building a proofof-concept system with the required functionality.

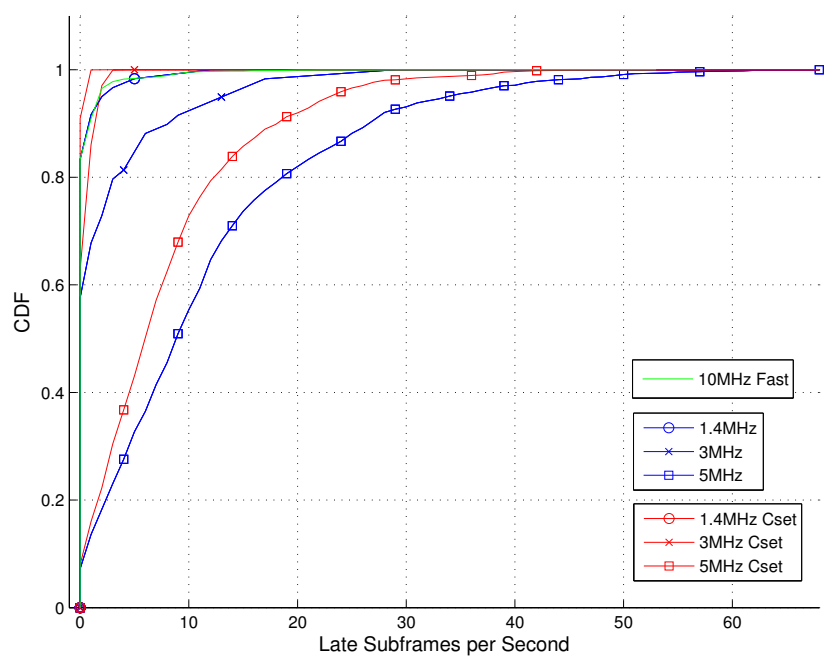

Figure 5: CDF of late subframes per second with E3-1230 and i7-4930K (Fast).

\section{CONCLUSIONS}

We propose a new architecture for implementing a SDR base station using general purpose processors and non-realtime operating system. In our architecture the Virtual Hardware Enhancement Layer VHEL maintains packet synchronization, sample synchronization and hides - to the extent feasible - the impact of lost or late BB packets. Late or lost packets appear as radio channel errors. Thanks to these functions we have effectively eliminated the strict real time requirements from $\mathrm{BB}$ operations. We have implemented a TD-LTE BS and UE using the proposed architecture and provided measurement results of the impact of using nonreal time operating system for $\mathrm{BB}$ processing. Our results show that with USRP N200 and normal PC it is possible to have a full TD-LTE implementation. We can also use the system as a simulator (emulator), by replacing the RRH with a simulated radio environment. This is useful especially when developing and testing the correct operation of complex radio resource control methods that impact multiple BB processes.

The proposed architecture opens up new possibilities for developing SDRAN. We have connected and synchronized multiple remote radio heads with a single BB processing unit. With this setup we can easily develop a simple massive MIMO testbed. We may also run multiple base stations in virtual machines in a server. The results of this research will be reported in future publications.

\section{ACKNOWLEDGMENTS}

We thank TEKES, the Finnish funding agency for innovation, for funding this work. The authors would also like to thank all the participants involved in the development process of our TD-LTE platform, especially Yihenew Beyene, Chen Guo and Gerardo Moreno Crespo.

\section{REFERENCES}

[1] 3GPP. Evolved Universal Terrestrial Radio Access (E-UTRA); Multiplexing and channel coding. TS 
36.212, 3rd Generation Partnership Project (3GPP), Sept. 2008.

[2] 3GPP. Evolved Universal Terrestrial Radio Access (E-UTRA); Physical channels and modulation. TS 36.211, 3rd Generation Partnership Project (3GPP), Sept. 2008.

[3] 3GPP. Evolved Universal Terrestrial Radio Access (E-UTRA); Physical layer procedures. TS 36.213, 3rd Generation Partnership Project (3GPP), Sept. 2008.

[4] Amarisoft. Amarisoft website. http://www. amarisoft.com/, 2014.

[5] ASGARD. ASGARD website. http://blog.asgard.lab.es.aau.dk/, 2014.

[6] J. Ellsberger, A. Sarma, and D. Hogrefe. SDL : formal object-oriented language for communicating systems. Prentice Hall, Harlow, UK, New York, Paris, 1997.

[7] Eurecom. Open Air Interface website. http://www.openairinterface.org/, 2014.

[8] GNURadio. GNURadio website. http://gnuradio.org/redmine/, 2014.

[9] H. Guan, T. Kolding, and P. Merz. Discovery of cloud-ran. In Cloud-RAN Workshop 2010, 2010.

[10] A. Gudipati, D. Perry, L. E. Li, and S. Katti. SoftRAN: Software Defined Radio Access Network. In Proceedings of the Second ACM SIGCOMM Workshop on Hot Topics in Software Defined Networking, HotSDN '13, pages 25-30, New York, NY, USA, 2013. ACM.

[11] V. Inc. VANU website. http://www.vanu.com/, 2014.

[12] J. Malsbury. Modular, open-source software transceiver for PHY/MAC research. In Proceedings of the Second Workshop on Software Radio Implementation Forum, SRIF '13, pages 31-36, New York, NY, USA, 2013. ACM.

[13] OpenBTS. OpenBTS website. http://openbts.org/, 2014.
[14] OpenLTE. OpenLTE website. http://openlte.sourceforge.net/, 2014.

[15] D. Sabella, P. Rost, Y. Sheng, E. Pateromichelakis, U. Salim, P. Guitton-Ouhamou, M. Di Girolamo, and G. Giuliani. RAN as a service: Challenges of designing a flexible RAN architecture in a cloud-based heterogeneous mobile network. In Future Network and Mobile Summit (FutureNetworkSummit), 2013, pages 1-8, July 2013.

[16] P. Sutton, J. Lotze, H. Lahlou, S. Fahmy, K. Nolan, B. Ozgul, T. Rondeau, J. Noguera, and L. Doyle. Iris: an architecture for cognitive radio networking testbeds. Communications Magazine, IEEE, 48(9):114-122, Sept 2010.

[17] X. Tao, Y. Hou, H. He, K. Wang, and Y. Xu. GPP-based soft base station designing and optimization (invited paper). In Communications and Networking in China (CHINACOM), 2012 7th International ICST Conference on, pages 49-53, Aug 2012.

[18] D. L. Tennenhouse and V. G. Bose. The SpectrumWare approach to wireless signal processing. Wireless Network Journal, 2, 1996.

[19] X. Zhang, J. Ansari, G. Yang, and P. Mahonen. TRUMP: Supporting efficient realization of protocols for cognitive radio networks. In New Frontiers in Dynamic Spectrum Access Networks (DySPAN), 2011 IEEE Symposium on, pages 476-487, May 2011.

[20] Z. Zhu, P. Gupta, Q. Wang, S. Kalyanaraman, Y. Lin, H. Franke, and S. Sarangi. Virtual Base Station pool: Towards a wireless network cloud for radio access networks. In Proceedings of the 8th ACM International Conference on Computing Frontiers, CF '11, pages 34:1-34:10, New York, NY, USA, 2011. ACM. 